\title{
Moving Horizon Control in Dynamic Games
}

\author{
W.A. van den Broek* \\ Tilburg University, Department of Econometrics \\ P.O. Box 90153 \\ 5000 LE Tilburg, The Netherlands \\ email:bram@kub.nl
}

\begin{abstract}
We consider a continuous time system influenced by different agents who adopt moving horizon control. The well known Nash equilibrium concept is used to define two solution concepts fitting in the moving horizon structure. One of them is analyzed in more detail in the class of linear quadratic games. The (dis)advantages of moving horizon control are illustrated by means of a government debt stabilization model.
\end{abstract}

Journal of Economic Literature Classification Numbers: C60, C72, C73 and E60.

Keywords: Moving Horizon Control and (LQ) Differential Games.

\section{Introduction}

Many developments in game and control theory in the last few decades have caused an increasing interest in using nonzero-sum dynamic games for modelling several economic problems. In particular in the area of environmental economics ([Ze91] or [Fe98]) or in the area of macro-economic policy coordination ([Aa95], [Ta86] or [Ne95]), dynamic game theory is a very natural framework to model problems. One of the basic questions that arises in these models deals with the information of the players, which can in real life be quite uncertain. Therefore, several solution concepts exist and the corresponding strategies are often compared with each other. Two

${ }^{*}$ I would like to thank J.C. Engwerda for giving me the idea of introducing moving horizon control in the area of dynamic game theory. I would also like to thank him and J.M. Schumacher for many valuable comments. 
well known solution concepts are the open loop (OL) and the feedback (FB) Nash equilibrium. The OL information structure is a bit shortcoming in its economic relevance. However, especially in the class of linear quadratic games, it is often possible to arrive at analytic results in this information structure. The FB information structure is more realistic, but one should expect fewer analytic results.

Another basic question arising in dynamic games concerns the planning horizon on which the players base their decisions. Is it finite or infinite? And, if it is finite, what is its length? These questions are not always easy to answer. In this paper we introduce new solution concepts in which finite and infinite horizon optimization are combined. As the name already reveals, in the so called moving horizon (MH) solution concepts the players continuously extend their horizon as time evolves, which allows them to incorporate new information of the system at any point of time. Obviously, feedback information is required in this approach, which make the MH solution concepts of practical importance. However, we will also make use of open loop notions leading to analytic results.

The origin of moving horizon control lies in the field of control theory, where it is also known under the names receding horizon control or model predictive control, often abbreviated as MPC. In the last twenty years MPC has become quite popular in industry. For some recent developments we refer to [Bit97] or to [Ni98]. Besides these references and many other literature on MPC in control theory there also exists literature in operations research resembling the moving horizon approach. For example in [Se91], one can find a theory about rolling horizon decision making which is mainly applied in production planning problems.

The outline of the paper is as follows. In the next section we define two new solution concepts based on moving horizon control. The first concept is based on the open loop Nash equilibrium and the second is based on the feedback Nash equilibrium. The paper mainly concentrates on the former concept. In section 3 and in the following sections we consider the class of linear quadratic games. For the open loop moving horizon solution we make use of the Hamiltonian approach. Special attention is paid to the scalar case. In section 4 we illustrate the theory of this paper with a government debt stabilization model, first introduced in [Ta86]. The paper ends with some concluding remarks.

\section{The Moving Horizon Solution Concept}

In traditional formulations of nonzero-sum differential games one has the choice between either a finite or an infinite horizon. It is not always clear what the length of the planning horizon should be. Why should the players limit their scope to a finite horizon? It is not to be expected that life ends after this period. Also infinite horizon models seem a bit shortcoming with respect to this point of view. What does it practically mean to consider a period of infinite length?

In principle it is to be expected that the players will base their actions on a finite hori- 
zon. However it is not to be expected that the players will not take into account the future after this horizon as time evolves. It is more likely that the players will extend their horizon during the evolution of the game. This is exactly the point of view in moving horizon control. At any point of time the players base their actions on a finite horizon and after a small period of time the players extend their horizon and re-optimize their actions based on the new information which has become available.

An important ingredient in differential games is the state evolution, influenced by the players through control functions in a way described by a differential equation:

$$
\dot{x}(t)=f\left(t, x(t), u_{1}(t), \cdots, u_{N}(t)\right), \quad x(0)=x_{0},
$$

where the state and the actions (or controls) of the players at time $t$ are denoted by $x(t) \in \mathbb{R}^{n}$ and $u_{i}(t) \in \mathbb{R}^{m_{i}}$, for $i=1, \cdots, N$, respectively. Furthermore, the initial state is denoted by $x_{0}$ and $\dot{x}$ is the time derivative of $x$. The costs of the players are defined on an infinite horizon. However, as pointed out before, the actions in moving horizon control will always be based on a finite horizon, say of length $L$. This leads us to introduce cost functionals, to be denoted by $\mathcal{L}_{i}^{t}$, defined on time periods of the form $[t, t+L]$ for all $t>0$ :

$$
\mathcal{L}_{i}^{t}\left(u_{1}, \cdots, u_{N}\right)=\int_{t}^{t+L} g_{i}\left(\tau, x(\tau), u_{1}(\tau), \cdots, u_{N}(\tau)\right) d \tau .
$$

The interpretation of these costs is as follows. At time $t$ functions $u_{i}$, defined on the interval $[t, t+L]$, are chosen by the players. Then from (1) a state $x$, also defined on the interval $[t, t+L]$, results. This state together with the controls of the players then lead to certain costs $\mathcal{L}_{i}^{t}$. We will refer to this finite horizon game on the interval $[t, t+L]$ as the local game at time $t$. Up to now, we have not spoken about the information structure of the game. In this paper we consider open loop and feedback information patterns, which both lead to different solution concepts. We are now able to define the two moving horizon solution concepts.

Definition 2.1 Let the set of controls $v_{i}^{t}:[t, t+L] \rightarrow \mathbb{R}^{m_{i}}$, for $i=1, \cdots, N$, constitute an open loop Nash equilibrium of the local game at time $t$. Then the set of controls $u_{i}(t):=v_{i}^{t}(t)$, for $i=1, \cdots, N$, is called an open loop moving horizon solution.

Definition 2.2 Let the set of controls $v_{i}^{t}:[t, t+L] \rightarrow \mathbb{R}^{m_{i}}$, for $i=1, \cdots, N$, be a realization of a feedback Nash equilibrium of the local game at time $t$. Then the set of controls $u_{i}(t):=v_{i}^{t}(t)$, for $i=1, \cdots, N$, is called a feedback moving horizon solution.

The two definitions differ in the information pattern adapted locally. Since the players need at any time $t$ the actual state of the system in order to arrive locally at an open loop or a feedback Nash equilibrium, the global information structure is of the feedback type, i.e. at any time $t$ the state $x(t)$ is known to the players. The players only use the initial value of their locally optimal controls and then at the next point of 
time they extend the horizon and again determine the initial optimal control values based on the extended horizon.

In the rest of this paper we will often use the abbreviations OLMH and FBMH to refer to the solution concepts defined in definition 2.1 and 2.2, respectively. We will also use these abbreviations as superscripts. So, for example $x^{O L M H}$ stands for the optimal state trajectory in an open loop moving horizon solution.

The OLMH and FBMH solution concepts involve a finite horizon problem with a moving interval, i.e. the local problem at time $t$. However, if the system is time invariant, i.e. if $f$ and $g_{i}$, for $i=1, \cdots, N$, do not explicitly depend on time, then this local problem can easily be rephrased on the fixed interval $[0, L]$. In the following lemma we describe this feature.

Lemma 2.3 Let $f$ and $g_{i}$, for $i=1, \cdots, N$, be time invariant. Consider the finite horizon differential game with state dynamics

$$
\dot{\xi}(\tau)=f\left(\xi(\tau), \nu_{1}(\tau), \cdots, \nu_{N}(\tau)\right), \quad \xi(0)=\xi_{0},
$$

and cost functionals

$$
\Lambda_{i}\left(\nu_{1}, \cdots, \nu_{N}\right)=\int_{0}^{L} g_{i}\left(\xi(\tau), \nu_{1}(\tau), \cdots, \nu_{N}(\tau)\right) d \tau,
$$

for $i=1, \cdots, N$. If the set of controls $\nu_{1}\left(\tau ; \xi_{0}\right), \cdots, \nu_{N}\left(\tau ; \xi_{0}\right)$ constitutes an open loop Nash equilibrium, then the set $t \mapsto \nu_{i}(0 ; x(t))$, for $i=1, \cdots, N$, is an OLMH solution. Similarly, if the set of controls $\nu_{1}\left(\tau ; \xi_{0}\right), \cdots, \nu_{N}\left(\tau ; \xi_{0}\right)$ realizes a feedback Nash equilibrium then the set $t \mapsto \nu_{i}(0, x(t))$, for $i=1, \cdots, N$, is a FBMH solution.

In the rest of this paper we will also refer to the game described by (3) and (4) as the local game at time $t$ or simply as the local game.

\section{Remarks:}

- In finite horizon problems the cost functionals are often provided with a terminal penalty, which is obviously not taken into account by the moving horizon solution concepts. Although it would not make the analysis more difficult if we would do so, it does not seem very realistic to do this in the moving horizon solution concepts, since the players will never reach the endpoint of the local game.

- Locally the players always play a finite horizon game (at least they play the initial step). However, the moving horizon solution concepts obviously lead to infinite horizon solutions, which may cause stability problems. So, besides existence and uniqueness questions, that naturally arise from the definition, another important issue is the stability, i.e. do moving horizon solutions stabilize the system? The length of the planning horizon in the local game $(L)$ plays an important role in this. It is to be expected (see also [Bit97]) that especially for small values of $L$ stability problems may arise. 
- An important notion which is often dealt with in the literature is time consistency, see e.g. [Ba89]. This notion deals with the incentive of the players to deviate from their strategies if there has been some unexpected change in the dynamics. Both moving horizon solution concepts are time consistent, since, by definition, at any point of time the actual state is used in the local game.

- It is important to realize that the moving horizon concepts are not defined as equilibria. It is simply a method to control the system which may resemble reality better than the control strategies based directly on the existing equilibrium concepts.

\section{Moving Horizon Control in LQ Games}

We will now concentrate on the class of 2-player linear quadratic (LQ) games, which are specified by a linear differential equation and quadratic cost functionals, i.e.

$$
\begin{aligned}
f\left(x, u_{1}, u_{2}\right) & =A x+B_{1} u_{1}+B_{2} u_{2}, \\
g_{1}\left(x, u_{1}, u_{2}\right) & =x^{T} Q_{1} x+u_{1}^{T} R_{11} u_{1}+u_{2}^{T} R_{12} u_{2}, \\
g_{2}\left(x, u_{1}, u_{2}\right) & =x^{T} Q_{2} x+u_{1}^{T} R_{21} u_{2}+u_{2}^{T} R_{22} u_{2},
\end{aligned}
$$

with $A, B_{1}, B_{2}, Q_{1}, Q_{2}, R_{11}, R_{12}, R_{21}$ and $R_{22}$ constant matrices of appropriate dimensions. Moreover, we assume that $Q_{1}, Q_{2}, R_{11}, R_{12}, R_{21}$ and $R_{22}$ are symmetric and that $Q_{i} \geq 0, R_{i i}>0$, for $i=1,2$. Without loss of generality we may assume that $R_{11}=R_{22}=I$. This can always be achieved by applying a regular transformation on the control spaces. In this section we will make use of the notations $S_{i}:=B_{i} B_{i}^{T}$, for $i=1,2, S_{01}:=B_{1} R_{21} B_{1}^{T}$ and $S_{02}:=B_{2} R_{12} B_{2}^{T}$.

\subsection{The OLMH Solution - General Formulation}

In this section we focus on the open loop moving horizon solution in LQ games. Our starting point is the result of lemma 2.3. It is well known (see e.g. [Ba95], chapter 6) that in the open loop Nash equilibria of LQ games the cross terms $\nu_{2}^{T} R_{12} \nu_{2}$ and $\nu_{1}^{T} R_{21} \nu_{1}$ (recall that we denoted state and controls by Greek symbols in the local game) play no role. Thus without loss of generality we assume in the rest of this section and in the next section that $R_{12}=R_{21}=0$.

In order to investigate the OLMH solution we need to consider the open loop Nash equilibrium of the local game. The Hamiltonian approach (see e.g. [Ba95], chapter 6, or [Eng98]) leads to an equivalent formulation of such an equilibrium in terms of a boundary value problem:

$$
\frac{d}{d \tau}\left[\begin{array}{c}
\xi(\tau) \\
\psi_{1}(\tau) \\
\psi_{2}(\tau)
\end{array}\right]=\Leftrightarrow M\left[\begin{array}{c}
\xi(\tau) \\
\psi_{1}(\tau) \\
\psi_{2}(\tau)
\end{array}\right], \quad \xi(0)=x(t), \quad \psi_{1}(L)=\psi_{2}(L)=0
$$


where we introduced the costate variables $\psi_{i}$, for $i=1,2$, and the matrix

$$
M:=\left[\begin{array}{ccc}
\Leftrightarrow A & S_{1} & S_{2} \\
Q_{1} & A^{T} & 0 \\
Q_{2} & 0 & A^{T}
\end{array}\right] .
$$

If the boundary value problem has a solution on the interval $[0, L]$, then the open loop Nash equilibrium is given by $\nu_{i}(\tau)=\Leftrightarrow B_{i}^{T} \psi_{i}(\tau)$. So, according to lemma 2.3 , the optimal controls in the OLMH solution can then be written as $u_{i}^{O L M H}(t)=\Leftrightarrow B_{i}^{T} \psi_{i}(0)$. Using this result we arrive at the following lemma.

\section{Lemma 3.1 Define}

$$
\left[\begin{array}{l}
H(L) \\
G_{1}(L) \\
G_{2}(L)
\end{array}\right]:=e^{M L}\left[\begin{array}{l}
I \\
0 \\
0
\end{array}\right],
$$

where $M$ is defined by (6). Then there exists a unique OLMH solution if and only if the matrix $H(L)$ is regular. Moreover, if this condition is satisfied, then the OLMH solution is unique and can in closed loop form be written as

$$
u_{i}^{O L M H}(t, L)=\Leftrightarrow B_{i}^{T} G_{i}(L) H^{-1}(L) x^{O L M H}(t, L), \quad t>0,
$$

for $i=1,2$.

Proof: The first part of the lemma, i.e. the existence condition, follows from theorem 1 in [Eng98]. For the second part, note that (5) implies that

$$
\left[\begin{array}{c}
\xi(L) \\
0 \\
0
\end{array}\right]=e^{-M L}\left[\begin{array}{c}
x(t) \\
\psi_{1}(0) \\
\psi_{2}(0)
\end{array}\right],
$$

or, equivalently,

$$
\left[\begin{array}{c}
H(L) \\
G_{1}(L) \\
G_{2}(L)
\end{array}\right] \xi(L)=\left[\begin{array}{c}
x(t) \\
\psi_{1}(0) \\
\psi_{2}(0)
\end{array}\right] .
$$

From this it follows that $\xi(L)=H^{-1}(L) x(t)$ and thus $\psi_{i}(0)=G_{i}(L) H^{-1}(L) x(t)$. This completes the proof.

Obviously, the parameter $L$ plays an important role in the OLMH solution. In the rest of this paper differentiation with respect to $L$ is denoted by a prime (in order to indicate the difference with differentiation with respect to $t$ ).

From (8) it follows that the optimal state trajectory in the OLMH solution satisfies the initial value problem

$$
\dot{x}^{O L M H}(t, L)=A_{c l}(L) x^{O L M H}(t, L), \quad x^{O L M H}(0, L)=x_{0},
$$


where the closed loop matrix $A_{c l}(L)$ is given by

$$
A_{c l}(L)=A \Leftrightarrow\left(S_{1} G_{1}(L)+S_{2} G_{2}(L)\right) H^{-1}(L) .
$$

This expression involves the matrix functions $G_{1}, G_{2}$ and $H$. They can either be derived by a direct computation of $e^{M L}$ or by solving the initial value problem

$$
\begin{aligned}
& H^{\prime}(L)=\Leftrightarrow A H(L)+S_{1} G_{1}(L)+S_{2} G_{2}(L), \quad H(0)=I, \\
& G_{i}^{\prime}(L)=Q_{i} H(L)+A^{T} G_{i}(L), \quad G_{i}(0)=0, \quad i=1,2 \text {. }
\end{aligned}
$$

Note that (11) and (12) indeed uniquely specify $H, G_{1}$ and $G_{2}$. A first question that needs to be answered is: Is $H(L)$ regular for all $L>0$ ? An obvious disadvantage of (11) is the coupling with the $G_{i}{ }^{\prime}$ s. We are not interested in the $G_{i}{ }^{\prime}$ 's if we want to investigate regularity of $H$. In the next theorem we obtain an equation that has only $H$ as unknown.

Theorem 3.2 If $A S_{i}$ is symmetric for $i=1,2$, then

$$
H^{\prime \prime}(L) \Leftrightarrow\left(A^{2}+S_{1} Q_{1}+S_{2} Q_{2}\right) H(L)=0, \quad H(0)=I, \quad H^{\prime}(0)=\Leftrightarrow A .
$$

Proof: Multiplying (12) with $S_{i}$, adding the resulting equations for $i=1,2$ and using the assumption $S_{i} A^{T}=A S_{i}$ yields

$$
S_{1} G_{1}^{\prime}+S_{2} G_{2}^{\prime}=\left(S_{1} Q_{1}+S_{2} Q_{2}\right) H+A\left(S_{1} G_{1}+S_{2} G_{2}\right) .
$$

According to (11), $S_{1} G_{1}+S_{2} G_{2}$ can be replaced by $H^{\prime}+A H$, which results in

$$
\Leftrightarrow A H^{\prime}+S_{1} G_{1}^{\prime}+S_{2} G_{2}^{\prime}=\left(A^{2}+S_{1} Q_{1}+S_{2} Q_{2}\right) H .
$$

Observe that the left hand side equals $H^{\prime \prime}$ (see (11)). Finally the initial conditions for $H$ and $H^{\prime}$ directly follow from the initial conditions in (11) and (12).

An obvious disadvantage of theorem 3.2 is the symmetry condition. On the other side, if this condition is satisfied then $H(L)$ is uniquely specified by (13), which enables us to compute $H$ without also having to compute $G_{1}$ and $G_{2}$. Moreover, in the next theorem we will show that the symmetry condition leads to a differential equation for $A_{c l}$ completely in terms of the original data.

Theorem 3.3 Let $H(L)$ be regular for all $L>0$. If $A S_{i}$ is symmetric for $i=1,2$, then

$$
A_{c l}^{\prime}(L)=A_{c l}^{2}(L) \Leftrightarrow\left(A^{2}+S_{1} Q_{1}+S_{2} Q_{2}\right) .
$$

Proof: Differentiating (10) and using (11) and (12) yields

$$
\begin{aligned}
A_{c l}^{\prime}= & \Leftrightarrow\left(S_{1} G_{1}^{\prime}+S_{2} G_{2}^{\prime}\right) H^{-1}+\left(S_{1} G_{1}+S_{2} G_{2}\right) H^{-1} H^{\prime} H^{-1}= \\
= & A_{c l}^{2} \Leftrightarrow\left(A^{2}+S_{1} Q_{1}+S_{2} Q_{2}\right)+\left(A S_{1} \Leftrightarrow S_{1} A^{T}\right) G_{1} H^{-1}+ \\
& +\left(A S_{2} \Leftrightarrow S_{2} A^{T}\right) G_{2} H^{-1}=A_{c l}^{2} \Leftrightarrow\left(A^{2}+S_{1} Q_{1}+S_{2} Q_{2}\right),
\end{aligned}
$$


where the latter equality follows by assumption.

Equation (14) together with the initial condition

$$
A_{c l}(0)=A
$$

uniquely specifies $A_{c l}$. The advantage is clear: only original data is needed to compute $A_{c l}$. The disadvantage is also clear: a rather strong symmetry condition needs to be satisfied.

A problem in computing $H, G_{1}$ and $G_{2}$ is clearly the number of unknowns. In the case $n=2$, the number of unknowns in (11) and (12) already equals 12 . Moreover in the $N$-player case the number of matrix differential equations obviously equals $N+1$. In the next theorem we reduce this number ( 3 in the 2-player case) in a special case to 2 .

Theorem 3.4 If $Q_{i}=q_{i} Q$, for some $q_{i} \in \mathbb{R}$ and some matrix $Q$, then $G_{i}(L)=q_{i} G(L)$, where $G$ is uniquely specified by

$$
G^{\prime}(L)=Q H(L)+A^{T} G(L), \quad G(0)=0 .
$$

Proof: Let $\tilde{G}_{i}(L)=q_{i} G(L)$, with $G(L)$ determined by (16), then $\tilde{G}_{i}^{\prime}(L)=Q_{i} H(L)+$ $A^{T} \tilde{G}_{i}(L)$ and $\tilde{G}_{i}(0)=0$. Obviously, $\tilde{G}$ satisfies the equations by which $G_{i}$ is uniquely specified, i.e. (12). But then it must hold that $G_{i}=\tilde{G}_{i}$.

We now describe a method to determine the optimal state and controls in the OLMH solution using the results of the theorems 3.3 and 3.4. Suppose that the conditions in these theorems are satisfied. Then $A_{c l}$ can be determined from (14) and (15). Due to the result of theorem 3.4, (10) can be rewritten as $G H^{-1}=\left(q_{1} S_{1}+q_{2} S_{2}\right)^{-1}\left(A \Leftrightarrow A_{c l}\right)$, provided that $q_{1} S_{1}+q_{2} S_{2}$ is regular. This then leads to the following expression for the optimal controls in the OLMH solution:

$$
u_{i}^{O L M H}(t, L)=\Leftrightarrow q_{i} B_{i}^{T}\left(q_{1} S_{1}+q_{2} S_{2}\right)^{-1}\left(A \Leftrightarrow A_{c l}(L)\right) x^{O L M H}(t, L),
$$

where the optimal state trajectory $x^{O L M H}$ is given by

$$
x^{O L M H}(t, L)=e^{A_{c l}(L) t} x_{0} .
$$

In the next section (about the scalar case) and in section 4 (an economic example about government debt stabilization) we will partly follow this method to arrive at explicit formulas for $x^{O L M H}$ and $u_{i}^{O L M H}$.

We end this section by considering the limit $L \rightarrow \infty$. This is the same as considering the limit of the open loop Nash equilibrium of the finite horizon game as the horizon length tends to infinity. In [Eng98] it is shown that if this limit exists and if the resulting equilibrium stabilizes the closed loop system, then the equilibrium that is obtained in the limit is also an open loop Nash equilibrium of the infinite horizon game. For the OLMH solution this result can be formulated as follows. 
Theorem 3.5 Let $H(L)$ be regular for all $L>0$. Suppose that $G_{i}(L) H^{-1}(L)$ has a limit for $L \rightarrow \infty$, for $i=1,2$. Let

$$
P_{i}:=\lim _{L \rightarrow \infty} G_{i}(L) H^{-1}(L), \quad i=1,2,
$$

and suppose that $A \Leftrightarrow S_{1} P_{1} \Leftrightarrow S_{2} P_{2}$ is stable. Then the OLMH solution converges to an open loop Nash equilibrium of the infinite horizon game as $L \rightarrow \infty$.

\subsection{The OLMH Solution - Scalar Case}

The model simplifies considerably if the system parameters are all scalars. In this case it can be shown that the OLMH solution exists and is unique for all $L>0$ and it is possible to derive explicit formulas for the optimal state and controls in the OLMH solution. This is done in appendix A, section A.1.

The optimal state trajectory is given by

$$
{ }_{x}^{O L M H}(t, L)= \begin{cases}e^{-w(L) t} x_{0}, & \text { if } s_{1} q_{1}+s_{2} q_{2}>0, \\ e^{a t} x_{0}, & \text { if } s_{1} q_{2}+s_{2} q_{2}=0,\end{cases}
$$

with

$$
w(L):=\mu \tanh (\mu L \Leftrightarrow \lambda),
$$

where $\mu$ and $\lambda$ are defined in (56) and (57) respectively. For stability in the case $s_{1} q_{1}+$ $s_{2} q_{2}>0$ it is necessary that $w(L)$ is positive. Let $\bar{L}:=\lambda / \mu$ (the unique root of the equation $w(L)=0)$. Then the following result is easily seen.

Theorem 3.6 If $s_{1} q_{1}+s_{2} q_{2}>0$, then if $a \leq 0$ the closed loop system is stable for all $L>0$ and if $a>0$ the closed loop system is unstable for $0<L \leq \bar{L}$ and stable for $L>\bar{L}$. If $s_{1} q_{1}+s_{2} q_{2}=0$, the closed loop system is stable if and only if $a<0$.

The behavior of optimal state trajectories (corresponding to stable closed loop systems and in the case $s_{1} q_{1}+s_{2} q_{2}>0$ ) is completely characterized by the following theorem and illustrated in figure 1.

Theorem 3.7 The function $t \mapsto x^{O L M H}(t, L)$ is strictly decreasing if $x_{0}>0$ and strictly increasing if $x_{0}<0$ for all $L>\max \{0, \bar{L}\}$. For any $L_{1}, L_{2}$ with $\max \{0, \bar{L}\}<L_{1}<$ $L_{2}$ we have $\left|\dot{x}^{O L M H}\left(0, L_{1}\right)\right|<\left|\dot{x}^{O L M H}\left(0, L_{2}\right)\right|$. Finally, there is no $t>0$ satisfying $x^{O L M H}\left(t, L_{1}\right)=x^{O L M H}\left(t, L_{2}\right)$ if $x_{0} \neq 0$.

Proof: The first and second part of the theorem are easily seen by computing $\dot{x}^{O L M H}$. For the third part, note that for $t>0$ the equations $x^{O L M H}\left(t, L_{1}\right)=x^{O L M H}\left(t, L_{2}\right)$ and $w\left(L_{1}\right)=w\left(L_{2}\right)$ are equivalent. The latter has no solution since $w$ is strictly increasing. 
In figure 1 the graphs of two optimal state trajectories in the OLMH solution are drawn in the case $x_{0}>0$. Note that for increasing $L$, the rate of convergence $w(L)$ is also increasing. For $L \rightarrow \infty$ this rate approaches $\Leftrightarrow \mu$, which is the rate of convergence corresponding to the open loop Nash equilibrium of the infinite horizon game (see theorem 3.5). Thus for each $L>0$ the OLMH solution converges more slowly to its steady state than the open loop Nash equilibrium of the infinite horizon game.

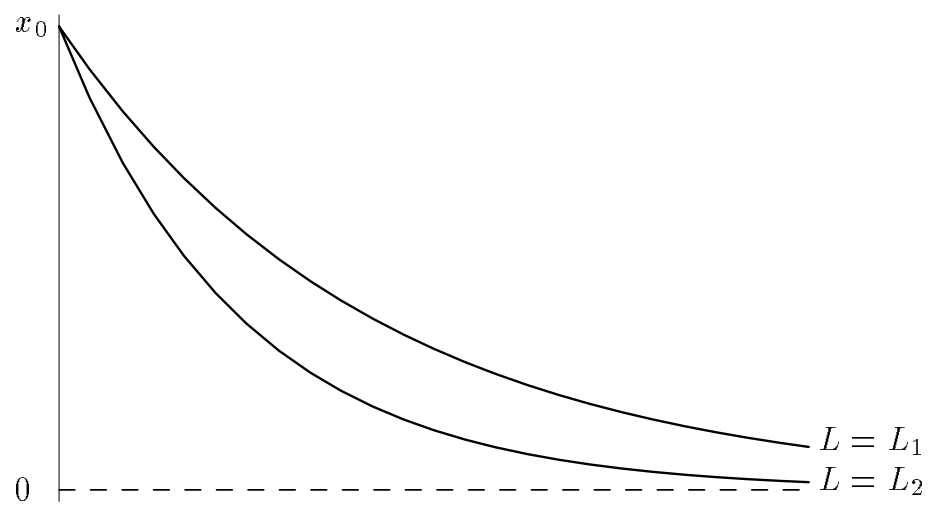

Figure 1: Optimal State in the OLMH Solution $\left(L_{1}<L_{2}\right)$.

Next we analyse the optimal controls in the OLMH solution. From section A.1 we conclude that

$$
u_{i}^{O L M H}(t, L)=\Leftrightarrow \frac{b_{i} q_{i}(w(L)+a) x_{0}}{s_{1} q_{1}+s_{2} q_{2}} e^{-w(L) t}, \quad \text { if } s_{1} q_{1}+s_{2} q_{2}>0,
$$

and $u_{i}^{O L M H}(t, L)=0$ if $s_{1} q_{1}+s_{2} q_{2}=0$. In the latter case the closed loop system obviously remains uncontrolled (this is of course not a big surprise!). The behavior of the optimal controls in the former case is characterized by the following theorem and illustrated in figure 2.

Theorem 3.8 The function $t \mapsto u_{i}^{O L M H}(t, L)$ is strictly increasing if $b_{i} x_{0}>0$ and strictly decreasing if $b_{i} x_{0}<0$ for all $L>\max \{0, \bar{L}\}$. For any $L_{1}, L_{2}$ with $\max \{0, \bar{L}\}<L_{1}<L_{2}$ we have

$$
0<u_{i}\left(0, L_{1}\right)<u_{i}\left(0, L_{2}\right)<\lim _{L \rightarrow \infty} u_{i}(0, L)=\Leftrightarrow \frac{b_{i} q_{i} x_{0}}{\mu \Leftrightarrow a}, \quad \text { if } b_{i} x_{0}<0,
$$

and

$$
\Leftrightarrow \frac{b_{i} q_{i} x_{0}}{\mu \Leftrightarrow a}=\lim _{L \rightarrow \infty} u_{i}(t, L)<u_{i}\left(0, L_{2}\right)<u_{i}\left(0, L_{1}\right)<0, \quad \text { if } b_{i} x_{0}>0 .
$$

Finally, there is one and only one $t>0$ satisfying $u_{i}^{O L M H}\left(t, L_{1}\right)=u_{i}^{O L M H}\left(t, L_{2}\right)$. 
Proof: First note that $w(0)=\Leftrightarrow a$ and thus $w(L)+a>0$ if $L>0$. The first part is now readily seen by computing $\dot{u}_{i}^{O L M H}$. The second part ((23) and (24)) follows from the monotonicity of $w$ and the fact that $w(L) \rightarrow \mu$ as $L \rightarrow \infty$. For the third part, note that the equation $u_{i}^{O L M H}\left(t, L_{1}\right)=u_{i}^{O L M H}\left(t, L_{2}\right)$ is equivalent to

$$
e^{\left(w\left(L_{2}\right)-w\left(L_{1}\right)\right) t}=\frac{w\left(L_{2}\right)+a}{w\left(L_{1}\right)+a}
$$

Since $w$ is strictly increasing, this equation has exactly one solution $t>0$.

In figure 2 two graphs of the optimal control in the OLMH solution of player $i$ in the case $b_{i} x_{0}>0$ are drawn. Let $t^{*}$ be the $t$-coordinate corresponding to the intersection point of the two graphs. By increasing $L$ from $L_{1}$ to $L_{2}$ the initial control increases. In fact it is readily seen from the figure that this property holds for all $t$ between 0 and $t^{*}$. For $t>t^{*}$ the situation is the other way around, i.e. by increasing $L$ from $L_{1}$ to $L_{2}$ the control value decreases. We conclude that in this sense the OLMH solution is better than the open loop Nash equilibrium of the infinite horizon game in the short run and worse in the long run.

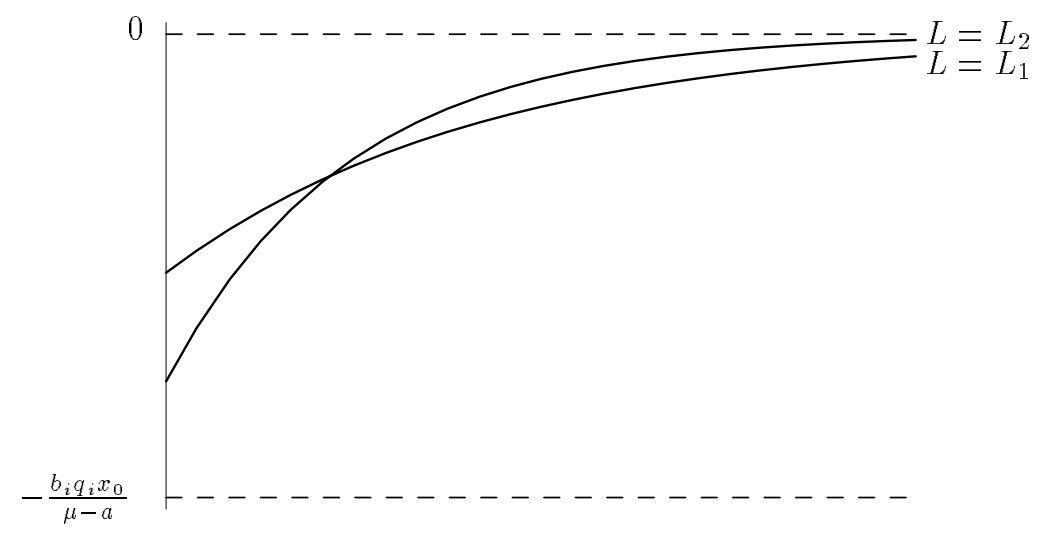

Figure 2: Optimal Control in the OLMH Solution $\left(L_{1}<L_{2}\right)$. 


\section{Government Debt Stabilization}

In this section we compute and analyse the OLMH solution in a model concerning government debt stabilization. This model has been introduced by Tabellini in [Ta86] and analyzed in a more general setting in [Aa95]. Our aim of applying moving horizon control to this economic situation is to show how one could use and analyze the moving horizon concept in a concrete situation. For more details concerning the economic interpretation we refer to [Aa95].

The model is a differential game in which the government debt is modeled as the state and the players are fiscal and monetary authorities. The (scalar) differential equation is given by

$$
\dot{d}(t)=r d(t)+f(t) \Leftrightarrow m(t), \quad d(0)=d_{0},
$$

with $d$ the government debt, $f$ the primary fiscal deficits, controlled by the fiscal authority, and $m$ the seignorage, controlled by the monetary authority. Furthermore, $r d(t)$ represents the interest payments on government debt and $d_{0}$ the initial stock of outstanding government debt. The objectives of the players are described by the cost functionals

$$
\mathcal{L}_{F}=\int_{0}^{\infty}\left((f(t) \Leftrightarrow \bar{f})^{2}+\eta(m(t) \Leftrightarrow \bar{m})^{2}+\kappa_{1}(d(t) \Leftrightarrow \bar{d})^{2}\right) \exp (\Leftrightarrow \delta t) d t
$$

and

$$
\mathcal{L}_{M}=\int_{0}^{\infty}\left((m(t) \Leftrightarrow \bar{m})^{2}+\kappa_{2}(d(t) \Leftrightarrow \bar{d})^{2}\right) \exp (\Leftrightarrow \delta t) d t
$$

respectively. The parameters $\eta, \kappa_{1}$ and $\kappa_{2}$ are weights, whereas $\bar{f}, \bar{m}$ and $\bar{d}$ are given target values. Furthermore $\delta$ is a discounting factor. All the parameters introduced so far, i.e. $r, d_{0}$, the weights, the targets and the discounting factor are assumed to be positive. Moreover we also assume that

$$
\begin{aligned}
d_{0} \Leftrightarrow \bar{d} & >0, \\
r \bar{d}+\bar{f} \Leftrightarrow \bar{m} & >0, \\
\delta \Leftrightarrow r & \geq 0, \\
\beta:=\kappa_{1}+\kappa_{2} \Leftrightarrow r(\delta \Leftrightarrow r) & >0 .
\end{aligned}
$$

Assumptions (28), (29) and (30) have been taken from [Aa95], section 2. The fourth assumption will turn out to be a necessary and sufficient condition for the existence of a specific horizon length (this value will be denoted by $\hat{L}$ ), with the property that the resulting closed loop system in the OLMH solution is stable if and only if $L>\hat{L}$. If (31) is violated the closed loop system is unstable for all $L>0$.

In section 3.1 we stressed the essential dependence of the OLMH solution with respect to $L$ by using $L$ as an argument in the state and control functions. We continue 
this convention in this section, i.e. $d=d(t, L), f=f(t, L)$ and $m=m(t, L)$. In order to write the model in the notation used in section 3.1, we introduce

$$
\begin{aligned}
x_{1}(t, L) & =(d(t, L) \Leftrightarrow \bar{d}) \exp (\Leftrightarrow \delta t / 2), \\
x_{2}(t) & =(r \bar{d}+\bar{f} \Leftrightarrow \bar{m}) \exp (\Leftrightarrow \delta t / 2), \\
u_{1}(t, L) & =(f(t, L) \Leftrightarrow \bar{f}) \exp (\Leftrightarrow \delta t / 2), \\
u_{2}(t, L) & =(m(t, L) \Leftrightarrow \bar{m}) \exp (\Leftrightarrow \delta t / 2) .
\end{aligned}
$$

Note that $x_{2}$ does indeed not depend on $L$. We have introduced two state variables, while the dynamics is in fact scalar. This is due to the presence of the target constants. In the following we denote the state as the column $x(t, L)=\left[\begin{array}{ll}x_{1}(t, L) & x_{2}(t)\end{array}\right]^{T}$. The initial condition is then given by $x(0, L)=\left[\begin{array}{ll}x_{10} & x_{20}\end{array}\right]^{T}$, with $x_{10}:=d_{0} \Leftrightarrow \bar{d}(>0)$ and $x_{20}:=r \bar{d}+\bar{f} \Leftrightarrow \bar{m}(>0)$. Next we introduce the matrices

$$
\begin{aligned}
& A=\left[\begin{array}{cc}
r \Leftrightarrow \frac{\delta}{2} & 1 \\
0 & \Leftrightarrow \frac{\delta}{2}
\end{array}\right], \quad B_{1}=\left[\begin{array}{l}
1 \\
0
\end{array}\right], \quad B_{2}=\left[\begin{array}{c}
\Leftrightarrow 1 \\
0
\end{array}\right], \\
& Q_{1}=\left[\begin{array}{cc}
\kappa_{1} & 0 \\
0 & 0
\end{array}\right], \quad Q_{2}=\left[\begin{array}{cc}
\kappa_{2} & 0 \\
0 & 0
\end{array}\right], \quad R_{12}=\eta \text {. }
\end{aligned}
$$

Note that there are no direct effects of the fiscal controls modeled in the monetary objectives, i.e. $R_{21}=0$. We already mentioned in section 3.1 that $R_{12}$ does not play a role in the OLMH solution. Thus in the remainder $\eta$ will not appear in any formula. This, in contrast to e.g. the FBMH solution, where $\eta$ would be an essential parameter.

In appendix A, section A.2, we first show that the OLMH solution exists for all $L>0$ and then we compute the OLMH solution. Here we analyse the resulting optimal state and controls in detail. We first focus on the resulting government debt dynamics. From (32), (74), (69), (70) and (72) we derive

$$
d^{O L M H}(t, L)=d_{\infty}(L)+\left(d_{0} \Leftrightarrow d_{\infty}(L)\right) e^{-h(L) t},
$$

with

$$
\begin{aligned}
h(L) & :=\alpha \tanh (\alpha L \Leftrightarrow \gamma) \Leftrightarrow \delta / 2, \\
d_{\infty}(L) & :=\bar{d}+\frac{x_{20}}{\beta}(\delta \Leftrightarrow r+\alpha \sqrt{\kappa} v(L)),
\end{aligned}
$$

where we defined

$$
v(L):=\frac{e^{-\delta L / 2}}{\alpha \sinh (\alpha L \Leftrightarrow \gamma) \Leftrightarrow \frac{\delta}{2} \cosh (\alpha L \Leftrightarrow \gamma)},
$$

and $\beta, \alpha, \kappa$ and $\gamma$ are given by (31), (59) and (71), respectively. The function $h$ is called the adjustment speed. Assumption (31) yields $\alpha>\delta / 2$, implying that there exists a unique zero of $h$, say $\hat{L}$. This $\hat{L}$ is defined by (73). It is not so difficult to see that $\hat{L}>0$. Since $h$ is strictly increasing, $h(L)$ is negative for $L<\hat{L}$ and positive for $L>\hat{L}$. Clearly, the system is only stable if the adjustment speed is positive. For 
that reason we assume throughout the rest of this section that $L>\hat{L}$. Obviously, for increasing $L$ the adjustment speed is also increasing and $h(L) \uparrow \alpha \Leftrightarrow \delta / 2$ as $L \rightarrow \infty$.

The assumption $L>\hat{L}$ ensures that $d_{\infty}(L)$ (see (38) and (39)) is well defined. Note that $d^{O L M H}(t, L) \rightarrow d_{\infty}(L)$ as $L \rightarrow \infty$ for all $L>\hat{L}$, justifying the notation $d_{\infty}$ for the steady state debt. In the next lemma and in theorem 4.2 a number of characteristic properties of $d^{O L M H}$ is obtained. See also figure 3 for an illustration of the characteristics of the government debt.

Lemma 4.1 If $x_{10} \Leftrightarrow(\delta \Leftrightarrow r) x_{20} / \beta \leq 0$, then $d_{\infty}(L)>d_{0}$ for all $L>\hat{L}$. If $x_{10} \Leftrightarrow(\delta \Leftrightarrow$ $r) x_{20} / \beta>0$, then there exists an $L^{*}>\hat{L}$ so that $d_{\infty}(L)>d_{0}$ for all $\hat{L}<L<L^{*}$, $d_{\infty}\left(L^{*}\right)=d_{0}$ and $d_{\infty}(L)<d_{0}$ for all $L>L^{*}$.

Proof: Because $h(L)>0$ for all $L>\hat{L}$, also $v(L)>0$ for all $L>\hat{L}$. Moreover, $v(L) \rightarrow \infty$ as $L \downarrow \hat{L}, v(L) \downarrow 0$ as $L \rightarrow \infty$ and $v$ is strictly decreasing for all $L>\hat{L}$. Now, consider

$$
d_{\infty}(L) \Leftrightarrow d_{0}=\Leftrightarrow\left(x_{10} \Leftrightarrow(\delta \Leftrightarrow r) x_{20} / \beta\right)+\alpha x_{20} \sqrt{\kappa} v(L) / \beta .
$$

If $x_{10} \Leftrightarrow(\delta \Leftrightarrow r) x_{20} / \beta \leq 0$, the right-hand side of this equation is positive for all $L>\hat{L}$, from which the first part of the theorem follows. Let $x_{10} \Leftrightarrow(\delta \Leftrightarrow r) x_{20} / \beta>0$. Then the right-hand side of the equation approaches $\infty$ as $L \downarrow \hat{L}$ and a negative number as $L \rightarrow \infty$ and it equals 0 for one and only one $L>\hat{L}$, say $L^{*}$. This proves the second part of the theorem.

Since $\bar{d}<d_{0}$ it is undesirable that the steady state debt exceeds the initial debt. Obviously (see lemma 4.1) it is possible to exclude this phenomenon by assuming that the parameters satisfy

$$
\zeta:=x_{10} \Leftrightarrow(\delta \Leftrightarrow r) x_{20} / \beta>0
$$

and to consider horizon lengths $L \geq L^{*}$. This is what we will do in the remainder of this section. Note that $L^{*}$ is the unique root of the equation $d_{\infty}(L)=d_{0}$ in the interval $(\hat{L}, \infty)$. Unfortunately, it is not possible to determine $L^{*}$ analytically. However it is not so difficult to do this numerically in concrete situations.

Theorem 4.2 The function $t \mapsto d^{O L M H}(t, L)$ is constant for $L=L^{*}$ and decreasing for $L>L^{*}$. For any $L_{1}, L_{2}$ with $L^{*}<L_{1}<L_{2}$ we have

$$
\bar{d}+x_{20}(\delta \Leftrightarrow r) / \beta=\lim _{L \rightarrow \infty} d_{\infty}(L)<d_{\infty}\left(L_{2}\right)<d_{\infty}\left(L_{1}\right)<d_{0} .
$$

Finally, the graphs of $t \mapsto d^{O L M H}\left(t, L_{1}\right)$ and $t \mapsto d^{O L M H}\left(t, L_{2}\right)$ do not intersect.

Proof: Taking the derivative of $d^{O L M H}$ with respect to $t$ and using the facts $d_{\infty}\left(L^{*}\right)=$ $d_{0}$ and $d_{\infty}(L)<d_{0}$ for all $L>L^{*}$ directly yields the first part of the theorem. The second part, i.e. (41), follows from assumption (30) and the basic observations concerning the function $v$ made at the beginning of the proof of the previous theorem. 
For the third part, assume there exists a $t_{0}>0$ with $d^{O L M H}\left(t_{0}, L_{1}\right)=d^{O L M H}\left(t_{0}, L_{2}\right)$. Then, according to the mean value theorem there must exist an $L_{3}$ with $L_{1}<L_{3}<L_{2}$ so that $\left(\partial d^{O L M H} / \partial L\right)\left(t_{0}, L_{3}\right)=0$, or, equivalently,

$$
d_{\infty}^{\prime}\left(L_{3}\right)\left(1 \Leftrightarrow e^{-h\left(L_{3}\right) t_{0}}\right) \Leftrightarrow\left(d_{0} \Leftrightarrow d_{\infty}\left(L_{3}\right)\right) h^{\prime}\left(L_{3}\right) t_{0} e^{-h\left(L_{3}\right) t_{0}}=0 .
$$

On the other hand, observe that the left-hand side of this equation is negative, which is obviously a contradiction.

In figure 3 four graphs of the optimal government debt in the OLMH solution are drawn. Note that $\lim _{L \rightarrow \infty} d_{\infty}(L)$ also equals $d_{0} \Leftrightarrow \zeta$ as depicted in this figure. Thus $\left[d_{0} \Leftrightarrow \zeta, d_{0}\right]$ indicates the range of the steady state debt. Furthermore, the gap between the target debt and the steady state debt exceeds $(\delta \Leftrightarrow r) x_{20} / \beta$ and for increasing $L$ this gap decreases. In this sense, the open loop Nash equilibrium of the infinite horizon game (this corresponds to the OLMH solution for $L \rightarrow \infty$, see theorem 3.5) is better than the OLMH solution for all $L \geq L^{*}$.

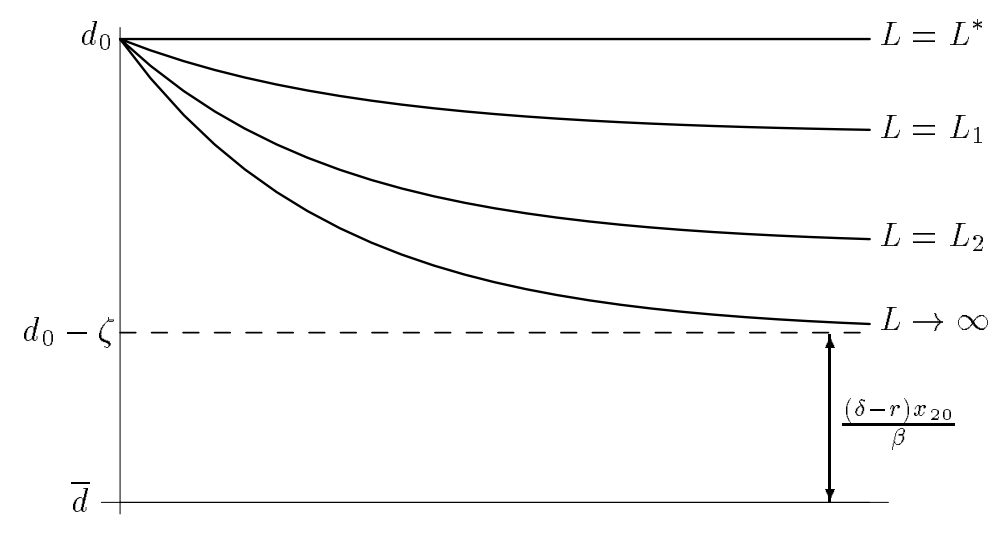

Figure 3: Government Debt in the OLMH Solution $\left(L_{1}<L_{2}\right)$.

Next we consider the optimal fiscal policy in the OLMH solution. From (34), (76), (74), (75), (69), (70) and (72) we derive

$$
f^{O L M H}(t, L)=f_{\infty}(L)+\left(f_{0}(L) \Leftrightarrow f_{\infty}(L)\right) e^{-h(L) t},
$$

with

$$
\begin{aligned}
f_{\infty}(L) & =\bar{f} \Leftrightarrow \frac{\kappa_{1} x_{20}}{\beta}\left(1+\frac{\alpha r}{\sqrt{\kappa}} v(L)\right), \\
f_{0}(L) & =\bar{f} \Leftrightarrow \frac{\kappa_{1}}{\kappa}\left(r x_{10}+x_{20}+\zeta h(L) \Leftrightarrow \frac{\alpha x_{20} \sqrt{\kappa} e^{-\delta L / 2}}{\beta \cosh (\alpha L \Leftrightarrow \gamma)}\right) .
\end{aligned}
$$


Recall that $\beta, \alpha, \kappa, \gamma$ and $\zeta$ are introduced by (31), (59), (71) and (40) and that we assumed $\beta>0$ and $\zeta>0$. Furthermore, $h(L)$ and $v(L)$ are introduced in (37) and (39). We only consider horizon lengths $L \geq L^{*}$, which makes $f_{0}$ and $f_{\infty}$ well defined. Since $h(L)>0$ we have $f^{O L M H}(0, L)=f_{0}(L)$ and $f^{O L M H}(t, L) \rightarrow f_{\infty}(L)$ as $t \rightarrow \infty$ for all $L \geq L^{*}$, justifying the notations $f_{0}$ and $f_{\infty}$. Let

$$
f^{*}:=\bar{f} \Leftrightarrow \frac{\kappa_{1}}{\kappa}\left(r x_{10}+x_{20}\right) .
$$

The results of the following theorem are illustrated in figure 4.

Theorem 4.3 The function $t \mapsto f^{O L M H}(t, L)$ is constant for $L=L^{*}$ (the constant value is $f^{*}$ ) and strictly increasing for $L>L^{*}$. Moreover, for any $L_{1}, L_{2}$ with $L^{*}<L_{1}<L_{2}$ we have

$$
f^{*} \Leftrightarrow \frac{\kappa_{1}}{\kappa} \zeta(\alpha \Leftrightarrow \delta / 2)=\lim _{L \rightarrow \infty} f_{0}(L)<f_{0}\left(L_{2}\right)<f_{0}\left(L_{1}\right)<f^{*}
$$

and

$$
f^{*}<f_{\infty}\left(L_{1}\right)<f_{\infty}\left(L_{2}\right)<\lim _{L \rightarrow \infty} f_{\infty}(L)=f^{*}+\frac{\kappa_{1}}{\kappa} \zeta r .
$$

Finally, there is one and only one $t>0$ satisfying $f^{O L M H}\left(t, L_{1}\right)=f^{O L M H}\left(t, L_{2}\right)$.

Proof: Since $d_{\infty}\left(L^{*}\right)=d_{0}$ we can derive an explicit expression for $v\left(L^{*}\right)$ from (38). Plugging this expression into (43) and (44), and using the identity

$$
e^{-\delta L / 2} / \cosh (\alpha L \Leftrightarrow \gamma)=v(L) h(L)
$$

yields $f_{0}\left(L^{*}\right)=f_{\infty}\left(L^{*}\right)=f^{*}$. This implies that $f^{O L M H}\left(t, L^{*}\right)=f^{*}$ for all $t \geq 0$.

Since $v$ is strictly decreasing it follows that $f_{\infty}$ is strictly increasing. Next, we analyse the monotonicity of $f_{0}$. The derivative of $f_{0}$ is given by

$$
f_{0}^{\prime}(L)=\Leftrightarrow \frac{\kappa_{1}}{\kappa} h^{\prime}(L)\left(\zeta \Leftrightarrow \frac{\alpha x_{20} \sqrt{\kappa}}{\beta} v(L)\right)+\frac{\kappa_{1} \alpha x_{20}}{\sqrt{\kappa} \beta} h(L) v^{\prime}(L) .
$$

In theorem 4.1 we showed $d_{\infty}<d_{0}$ for $L>L^{*}$. This inequality is equivalent to $\zeta \Leftrightarrow x_{20} \alpha \sqrt{\kappa} v(L) / \beta>0$, implying that $f_{0}$ is strictly decreasing. The fact that $f_{0}\left(L^{*}\right)=$ $f_{\infty}\left(L^{*}\right)$ combined with the monotonicity properties of $f_{0}$ and $f_{\infty}$ implies that $f_{0}(L)<$ $f_{\infty}(L)$ for all $L>L^{*}$. From this we conclude that $\left(\partial f^{O L M H} / \partial t\right)(t, L)=\Leftrightarrow\left(f_{0}(L) \Leftrightarrow\right.$ $\left.f_{\infty}(L)\right) h(L) e^{-h(L) t}>0$, which completes the first part of the theorem. The second part of the theorem consists of (46) and (47). The limits for $L \rightarrow \infty$ directly follow from $v(L) \rightarrow 0, h(L) \rightarrow \alpha \Leftrightarrow \delta / 2$ as $L \rightarrow \infty$. The other assertions in (46) and (47) follow from the proof of the first part of this theorem. For the third part, let $F(t)=$ $f^{O L M H}\left(t, L_{1}\right) \Leftrightarrow f^{O L M H}\left(t, L_{2}\right)$. Then obviously $F(0)>0$ and $\lim _{t \rightarrow \infty} F(t)<0$, showing the existence of a $t$ with the desired property. For the uniqueness, consider the equation $\dot{F}(t)=0$, or, equivalently

$$
e^{\left(h\left(L_{2}\right)-h\left(L_{1}\right)\right) t}=\frac{h\left(L_{2}\right)\left(f_{\infty}\left(L_{2}\right) \Leftrightarrow f_{0}\left(L_{2}\right)\right)}{h\left(L_{1}\right)\left(f_{\infty}\left(L_{1}\right) \Leftrightarrow f_{0}\left(L_{1}\right)\right)} .
$$


Since $h\left(L_{2}\right)\left(f_{\infty}\left(L_{2}\right) \Leftrightarrow f_{0}\left(L_{2}\right)\right)>h\left(L_{1}\right)\left(f_{\infty}\left(L_{1}\right) \Leftrightarrow f_{0}\left(L_{1}\right)\right)>0$ and $h\left(L_{2}\right)>h\left(L_{1}\right)$ we observe that this equation has exactly one solution. This indicates that there also exists a unique $t>0$ satisfying the equation $F(t)=0$.

Note that the limit of $f_{\infty}$ for $L \rightarrow \infty$ can also be written as

$$
\lim _{L \rightarrow \infty} f_{\infty}(L)=\bar{f} \Leftrightarrow \frac{\kappa_{1} x_{20}}{\beta} .
$$

The limits in (46) and (47) are written in such a format that the margins for the initial $(t=0)$ and asymptotic $(t \rightarrow \infty)$ fiscal control, i.e. $\kappa_{1} \zeta(\alpha \Leftrightarrow \delta / 2) / \kappa_{1}$ and $\kappa_{1} \zeta r / \kappa_{1}$ respectively, are directly visible and easily comparable. The format in (48) reveals the extra information that the target value $\bar{f}$ is never reached and that the gap between the asymptotic fiscal control and the target is at least $\kappa_{1} x_{20} / \beta$.

Theorem 4.3 implies that for any $L>L^{*}$ there exists exactly one $t>0$ satisfying the equation $f^{O L M H}(t, L)=f^{*}$. This enables us to define a function $\psi$ on $\left(L^{*}, \infty\right)$ implicitly by

$$
t=\psi(L): \Leftrightarrow f^{O L M H}(t, L)=f^{*} .
$$

Thus, the point $\left(\psi(L), f^{*}\right)$ is the intersection point of the curves corresponding to the equations $f=f(t, L)$ and $f=f^{*}$ in the $(t, f) \Leftrightarrow$ plane. The equation $f^{O L M H}(t, L)=$ $f^{*}$ can be written as

$$
(r+h(L))\left(\zeta \Leftrightarrow \frac{x_{20} \alpha \sqrt{\kappa}}{\beta} v(L)\right) e^{-h(L) t}=r\left(\zeta \Leftrightarrow \frac{x_{20} \alpha \sqrt{\kappa}}{\beta} v(L)\right) .
$$

Now, $L>L^{*}$ implies that $\zeta \Leftrightarrow x_{20} \alpha \sqrt{\kappa} v(L) / \beta>0$ (see also the proof of theorem 4.3) and this factor is therefore unequal to 0 . Consequently, $\psi$ is given by

$$
\psi(L)=\frac{1}{h(L)} \log \left(1+\frac{h(L)}{r}\right), \quad L>L^{*} .
$$

It can easily be seen that $\psi$ is bounded and decreasing. The upperbound is reached for $L \downarrow L^{*}$ and can easily be determined numerically in concrete situations, whereas the lowerbound (reached for $L \rightarrow \infty)$ is given by $(\alpha \Leftrightarrow \delta / 2)^{-1} \log (1+(\alpha \Leftrightarrow \delta / 2) / r)$.

With the results of theorem 4.3 and the discussion about $\psi$ we can visualize the behavior of the fiscal control in the OLMH solution, see figure 4. As mentioned before, the gap between the asymptotic fiscal control ands the target exceeds $\kappa_{1} x_{20} / \beta$ for all $L \geq L^{*}$ and for increasing $L$ this gap decreases. In this sense, the open loop Nash equilibrium of the infinite horizon game is better than the OLMH solution. However, the gap between the initial fiscal control and the target is increasing for increasing $L$. In fact by increasing $L$ from $L_{1}$ to $L_{2}$ the gap between the optimal fiscal control and the target increases for all $t$ between 0 and $t^{*}$, where $t^{*}$ is the $t$-coordinate corresponding to the intersection point of the curves $f=f\left(t, L_{1}\right)$ and $f=f\left(t, L_{2}\right)$. In this sense, the OLMH solution is better than the open loop Nash equilibrium of the infinite horizon game in the short run, whereas it is the other way around in the long run. 


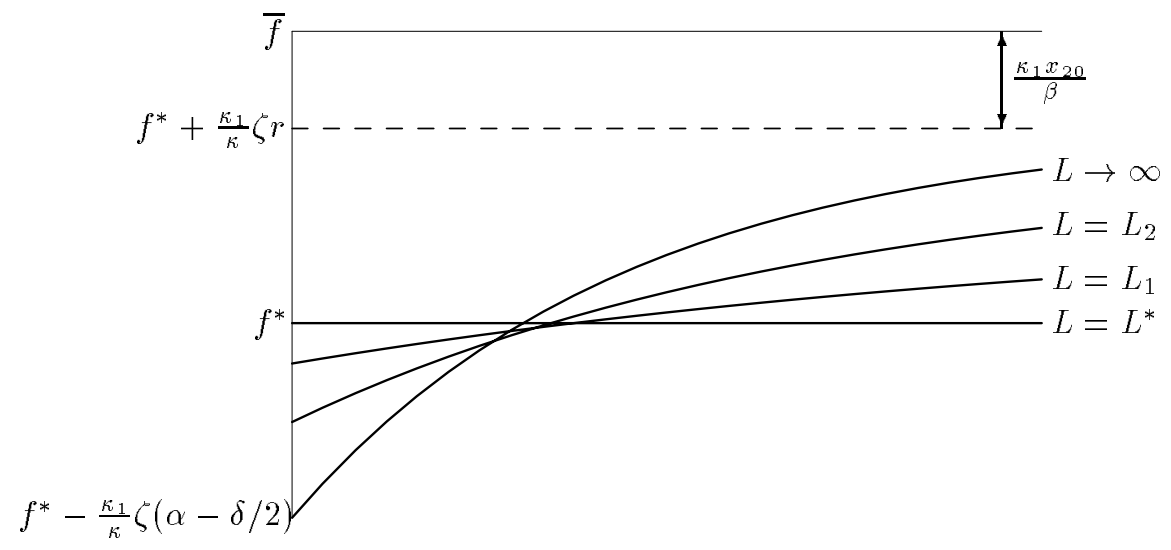

Figure 4: Fiscal Control in the OLMH Solution $\left(L_{1}<L_{2}\right)$.

We proceed by analyzing the monetary policy in the OLMH solution. Since the optimal controls only differ by a multiplicative constant (see (77)), the results of the optimal fiscal control can easily be transformed into results for the optimal monetary control. From (34), (35) and (77) it follows that

$$
m^{O L M H}(t, L)=\bar{m} \Leftrightarrow \frac{\kappa_{2}}{\kappa_{1}}\left(f^{O L M H}(t, L) \Leftrightarrow \bar{f}\right) .
$$

Let $m_{0}(L):=m^{O L M H}(0, L), m_{\infty}(L):=\lim _{t \rightarrow \infty} m^{O L M H}(t, L)$ and

$$
m^{*}:=\bar{m}+\frac{\kappa_{2}}{\kappa}\left(r x_{10}+x_{20}\right) \text {. }
$$

The following theorem then directly follows from theorem 4.3.

Theorem 4.4 The function $t \mapsto m^{O L M H}(t, L)$ is constant for $L=L^{*}$ (the constant value is $m^{*}$ ) and strictly decreasing for $L>L^{*}$. Moreover, for any $L_{1}, L_{2}$ with $L^{*}<L_{1}<L_{2}$ we have

$$
m^{*}<m_{0}\left(L_{1}\right)<m_{0}\left(L_{2}\right)<\lim _{L \rightarrow \infty} m_{0}(L)=m^{*}+\frac{\kappa_{2}}{\kappa} \zeta(\alpha \Leftrightarrow \delta / 2)
$$

and

$$
m^{*} \Leftrightarrow \frac{\kappa_{2}}{\kappa} \zeta r=\lim _{L \rightarrow \infty} m_{\infty}(L)<m_{\infty}\left(L_{2}\right)<m_{\infty}\left(L_{1}\right)<m^{*} .
$$

Finally, there is one and only one $t>0$ satisfying $m^{O L M H}\left(t, L_{1}\right)=m^{O L M H}\left(t, L_{2}\right)$.

The limit of $m_{\infty}$ for $L \rightarrow \infty$ can also be written as $\bar{m}+\kappa_{2} x_{20} / \beta$. This implies that the gap between the asymptotic monetary control and the target is at least $\kappa_{2} x_{20} / \beta$. 
Finally, note that the equation $f^{O L M H}(t, L)=f^{*}$ is equivalent to $m^{O L M H}(t, L)=m^{*}$ so that the point $\left(\psi(L), m^{*}\right)$ is the intersection point between the curves corresponding to $m=m(t, L)$ and $m=m^{*}$ in the $(t, m) \Leftrightarrow$ plane.

We visualize the behavior of the monetary control in the OLMH solution in figure 5. In a similar manner as for the fiscal player it can be argued that also for the monetary player the OLMH solution is more interesting than the open loop Nash equilibrium of the infinite horizon game in the short run and that the latter is more interesting in the long run.

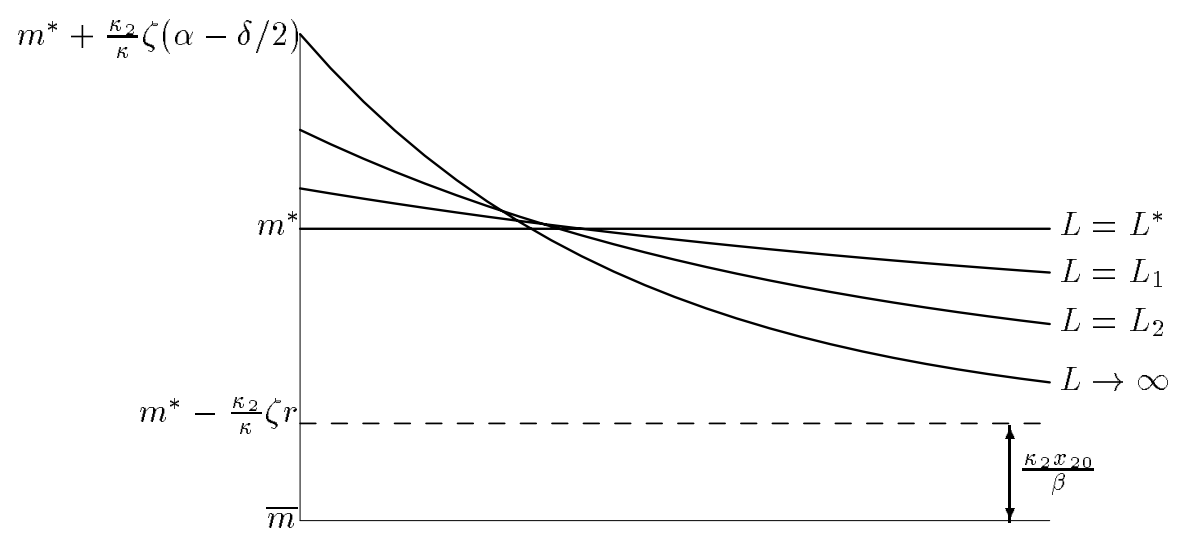

Figure 5: Monetary Control in the OLMH Solution $\left(L_{1}<L_{2}\right)$.

\section{Concluding Remarks}

In this paper the concept of moving horizon control has been introduced in the area of nonzero-sum differential games. Specifically, we defined two solution concepts (based on either open loop or feedback Nash equilibria) combining both finite and infinite horizon optimization. At any point of time the players determine an optimal control based on a finite horizon of length $L$ (the local game) and only play the initial control. This is repeated continuously in time and in this way a control defined on an infinite horizon results. If the local game is played with an open loop Nash equilibrium the resulting strategy is called an open loop moving horizon (OLMH) solution. Similarly, locally playing a feedback Nash equilibrium is called a feedback moving horizon (FBMH) solution. It is important to realize that both the OLMH and FBMH solutions are not equilibria. It is simply a method to control the system, which may be more in line with the paradigm of bounded rationality than the existing equilibrium concepts on finite or infinite horizons.

The OLMH solution concept has been further analyzed in linear quadratic games. In 
this setting we stressed the fact that the resulting optimal state and controls typically depend on $L$ and that this solution converges to the open loop Nash equilibrium of the infinite horizon game as $L \rightarrow \infty$. We showed that if the OLMH solution exists, it is unique. In section 3.1 we also treated a number of theorems which can be used for analyzing the existence and the stability if the original data satisfies certain conditions.

In the scalar case and in the economic example concerning government debt stabilization the OLMH solution has been determined analytically. In both cases the rate of convergence of the optimal state increases for increasing $L$ and in this sense the open loop Nash equilibrium is always preferable to the OLMH solution. Moreover for small values of $L$ the closed loop system can become unstable. Still it might be interesting to play a game in the OLMH solution. Apart from its conceptual advantages, there is another advantage in the resulting optimal control. For decreasing $L$ the gap between the initial control and the target control (0 in the scalar case) also decreases. Thus for smaller values of $L$ (of course then also stability problems may arise) the controls are initially closer to their targets. Asymptotically however, the situation is different, i.e. for decreasing $L$ the gap between control and target increases for large $t$. In this sense the OLMH solution is preferable to the open loop Nash equilibrium of the infinite horizon game in the short run and in the long run it is the other way around as should be expected.

\section{A Derivation of Optimal State and Controls}

In the next two sections we derive the optimal state trajectory and controls in the open loop moving horizon solution for the scalar case, treated in section 3.2, and the economic example of section 4.

\section{A.1 The Scalar Case}

The system parameters are $A=a, B_{i}=b_{i}$ and $Q_{i}=q_{i}$ with $a, b_{i}, q_{i} \in \mathbb{I R}$ and moreover $q_{i} \geq 0$. Note that also $s_{i}\left(=S_{i}\right)=b_{i}^{2} \geq 0$. Let

$$
\mu:=\sqrt{a^{2}+s_{1} q_{1}+s_{2} q_{2}}
$$

then, according to theorem 3.2 (note that the conditions are trivially satisfied), $H$ satisfies $H^{\prime \prime} \Leftrightarrow \mu^{2} H=0(L>0), H(0)=1$ and $H^{\prime}(0)=\Leftrightarrow a$. Consequently, $H(L)=$ $\cosh (\mu L) \Leftrightarrow(a / \mu) \sinh (\mu L)$ if $\mu>0$ and $H(L)=1$ if $\mu=0$ (note that this implies that also $a=0$ ). In both cases we have $H(L) \neq 0$ for all $L \geq 0$ and so, lemma 3.1 yields the existence of a unique OLMH solution for all $L \geq 0$.

The next step is the computation of $a_{c l}\left(=A_{c l}\right)$ by means of the result of theorem 3.3 (note that the conditions are satisfied). According to this theorem, $a_{c l}$ satisfies $a_{c l}^{\prime}=$ 
$a_{c l}^{2} \Leftrightarrow \mu^{2}(L>0)$ and $a_{c l}(0)=a$. Consequently, $a_{c l}(L)=\Leftrightarrow \mu \tanh (\mu L \Leftrightarrow \lambda)$, with

$$
\lambda:=\frac{1}{2} \log \frac{\mu+a}{\mu \Leftrightarrow a},
$$

if $s_{1} q_{1}+s_{2} q_{2}>0$ and $a_{c l}(L)=a$ if $s_{1} q_{1}+s_{2} q_{2}=0$.

For the computation of the optimal controls in the OLMH solution we follow the method described below theorem 3.4. Note that the conditions of this theorem are satisfied. It follows immediately that the optimal controls are given by (17) if $s_{1} q_{1}+$ $s_{2} q_{2}>0$.

If $s_{1} q_{1}+s_{2} q_{2}=0$, then also $s_{1} q_{1}=s_{2} q_{2}=0$. Now, from (8) and theorem 3.4, we observe that the optimal controls contain the factor $b_{i} q_{i}= \pm \sqrt{s_{i}} q_{i}=0$. Obviously the optimal controls are zero in the case $s_{1} q_{1}+s_{2} q_{2}=0$.

\section{A.2 Government Debt Stabilization}

Recall from section 4 that

$$
\begin{aligned}
A & =\left[\begin{array}{cc}
r \Leftrightarrow \frac{\delta}{2} & 1 \\
0 & \Leftrightarrow \frac{\delta}{2}
\end{array}\right], & B_{1}=\left[\begin{array}{l}
1 \\
0
\end{array}\right], & B_{2}=\left[\begin{array}{c}
\Leftrightarrow 1 \\
0
\end{array}\right], \\
Q_{1} & =\kappa_{1}\left[\begin{array}{ll}
1 & 0 \\
0 & 0
\end{array}\right], & Q_{2} & =\kappa_{2}\left[\begin{array}{ll}
1 & 0 \\
0 & 0
\end{array}\right],
\end{aligned}
$$

with $r, \delta, \kappa_{1}, \kappa_{2}>0$. It is also assumed that $\beta\left(=\kappa_{1}+\kappa_{2} \Leftrightarrow r(\delta \Leftrightarrow r)\right)$ is positive (see (31)). Since

$$
S_{1}=S_{2}=\left[\begin{array}{ll}
1 & 0 \\
0 & 0
\end{array}\right]
$$

it follows that $A S_{1}\left(=A S_{2}\right)$ is symmetric, so that the conditions in theorem 3.2 are satisfied. From this theorem it then follows that $H$ satisfies the second order differential equation (13). Write $H=\left[\begin{array}{ll}h_{11} & h_{12} \\ h_{21} & h_{22}\end{array}\right]$, then, since

$$
A^{2}+S_{1} Q_{1}+S_{2} Q_{2}=\left[\begin{array}{cc}
\alpha^{2} & r \Leftrightarrow \delta \\
0 & \delta^{2} / 4
\end{array}\right]
$$

with

$$
\alpha:=\sqrt{(r \Leftrightarrow \delta / 2)^{2}+\kappa}, \quad \kappa:=\kappa_{1}+\kappa_{2},
$$

$h_{11}, h_{12}, h_{21}$ and $h_{22}$ satisfy

$$
\begin{array}{lll}
h_{11}^{\prime \prime}=\alpha^{2} h_{11}+(r \Leftrightarrow \delta) h_{21}, & h_{11}(0)=1, & h_{11}^{\prime}(0)=\Leftrightarrow(r \Leftrightarrow \delta / 2), \\
h_{12}^{\prime \prime}=\alpha^{2} h_{12}+(r \Leftrightarrow \delta) h_{22}, & h_{12}(0)=0, & h_{12}^{\prime}(0)=\Leftrightarrow 1, \\
h_{21}^{\prime \prime}=\left(\delta^{2} / 4\right) h_{21}, & h_{21}(0)=0, & h_{21}^{\prime}(0)=0, \\
h_{22}^{\prime \prime}=\left(\delta^{2} / 4\right) h_{22}, & h_{22}(0)=1, & h_{22}^{\prime}(0)=\delta / 2,
\end{array}
$$


From (62) and (63) it follows that $h_{21}(L)=0$ and $h_{22}(L)=\exp (\delta L / 2)$, for all $L>0$. This reduces (60) to $h_{11}^{\prime \prime}=\alpha^{2} h_{11}$ and thus $\left.h_{11}(L)=\cosh (\alpha L) \Leftrightarrow(r \Leftrightarrow \delta / 2) / \alpha\right) \sinh (\alpha L)$. We conclude that

$$
\operatorname{det} H(L)=\left(\cosh (\alpha L) \Leftrightarrow \frac{r \Leftrightarrow \delta / 2}{\alpha} \sinh (\alpha L)\right) \exp (\delta L / 2)
$$

Since $|r \Leftrightarrow \delta / 2|<\alpha$, it follows that $\operatorname{det} H(L) \neq 0$, for all $L>0$. So, obviously, the conditions of theorem 3.3 are also satisfied, implying that the closed loop matrix is uniquely specified by (14) and (15). Write $A_{c l}=\left[\begin{array}{ll}a_{1} & a_{2} \\ a_{3} & a_{4}\end{array}\right]$, then $a_{1}, \cdots, a_{4}$ satisfy

$$
\begin{aligned}
& a_{1}^{\prime}=a_{1}^{2}+a_{2} a_{3} \Leftrightarrow \alpha^{2}, \quad a_{1}(0)=r \Leftrightarrow \delta / 2, \\
& a_{2}^{\prime}=a_{2}\left(a_{1}+a_{4}\right) \Leftrightarrow(r \Leftrightarrow \delta), \quad a_{2}(0)=1, \\
& a_{3}^{\prime}=a_{3}\left(a_{1}+a_{4}\right), \quad a_{3}(0)=0, \\
& a_{4}^{\prime}=a_{2} a_{3}+a_{4}^{2} \Leftrightarrow \delta^{2} / 4, \quad a_{4}(0)=\Leftrightarrow \delta / 2 .
\end{aligned}
$$

It is immediately clear that $a_{3}(L)=0$, which reduces (65) and (68) to $a_{1}^{\prime}=a_{1}^{2} \Leftrightarrow \alpha^{2}$ and $a_{4}^{\prime}=a_{4}^{2} \Leftrightarrow \delta^{2} / 4$, respectively. Together with the initial conditions for $a_{1}$ and $a_{4}$ it then follows that

$$
\begin{aligned}
& a_{1}(L)=\Leftrightarrow \alpha \tanh (\alpha L \Leftrightarrow \gamma), \\
& a_{4}(L)=\Leftrightarrow \frac{\delta}{2},
\end{aligned}
$$

with

$$
\gamma:=\frac{1}{2} \log \frac{\alpha+r \Leftrightarrow \delta / 2}{\alpha \Leftrightarrow r+\delta / 2}
$$

(due to the fact that $|r \Leftrightarrow \delta / 2|<\alpha$ it follows that the argument of the logarithm is positive, so that $\gamma$ is well defined). Substituting the expressions for $a_{1}$ and $a_{4}$ in (66) and solving the resulting differential equation yields

$$
a_{2}(L)=\frac{1}{\beta}\left((r \Leftrightarrow \delta)\left(\frac{\delta}{2} \Leftrightarrow \alpha \tanh (\alpha L \Leftrightarrow \gamma)\right)+\frac{\alpha \sqrt{\kappa} e^{-\delta L / 2}}{\cosh (\alpha L \Leftrightarrow \gamma)}\right) .
$$

The optimal state trajectory is the solution of the differential equations $\dot{x}_{1}=a_{1} x_{1}+$ $a_{2} x_{2}$ and $\dot{x}_{2}=a_{4} x_{2}$. Clearly, the case $a_{1}=a_{4}$ needs to be considered separately. Since $\alpha^{2} \Leftrightarrow \delta^{2} / 4=\beta>0$, it follows that $\alpha>\delta / 2$. This implies that there exists a unique $L$, say $\hat{L}$, so that $a_{1}(\hat{L})=a_{4}(\hat{L})$. It is easily seen that

$$
\hat{L}=\frac{1}{2 \alpha} \log \frac{(\alpha+r \Leftrightarrow \delta / 2)(\alpha+\delta / 2)}{(\alpha \Leftrightarrow(r \Leftrightarrow \delta / 2))(\alpha \Leftrightarrow \delta / 2)}>0
$$

For stability reasons (for details see section 4), we consider the functions $a_{1}, a_{2}, a_{3}$ and $a_{4}$ only for $L>\hat{L}$. This implies that $a_{1}(L)<a_{4}(L)$ and in particular that $a_{1}(L) \neq$ $a_{4}(L)$. The optimal state trajectory is then given by

$$
\begin{aligned}
x_{1}^{O L M H}(t, L) & =\frac{a_{2}(L) x_{20}}{a_{4}(L) \Leftrightarrow a_{1}(L)}\left(e^{a_{4}(L) t} \Leftrightarrow e^{a_{1}(L) t}\right)+x_{10} e^{a_{1}(L) t}, \\
x_{2}^{O L M H}(t, L) & =x_{20} e^{a_{4}(L) t} .
\end{aligned}
$$


We proceed by deriving the optimal controls. Note that the conditions of theorem 3.4 are satisfied, so that $G_{i}=\kappa_{i} G$, where $G$ follows from (16). This reduces (8) to

$$
u_{i}^{O L M H}(t, L)=\Leftrightarrow \varkappa_{i} B_{i}^{T} G(L) H^{-1}(L) x^{O L M H}(t, L) .
$$

Note that both the $B_{i}$ 's have a zero on the second row, implying that we only need the first row of $G H^{-1}$. From (10) we derive that

$$
G H^{-1}=\left[\begin{array}{cc}
\frac{r-(\delta / 2)-a_{1}}{\kappa} & \frac{1-a_{2}}{\kappa} \\
* & *
\end{array}\right]
$$

and hence

$$
\begin{aligned}
& u_{1}^{O L M H}(t, L)=\frac{\kappa_{1}}{\kappa}\left(\left(a_{1}(L) \Leftrightarrow(r \Leftrightarrow \delta / 2)\right) x_{1}^{O L M H}(t, L)+\left(a_{2}(L) \Leftrightarrow 1\right) x_{2}^{O L M H}(t, L)\right) \\
& u_{2}^{O L M H}(t, L)=\Leftrightarrow \frac{\kappa_{2}}{\kappa_{1}} u_{1}^{O L M H}(t, L) .
\end{aligned}
$$




\section{References}

[Aa95] Aarle B. van, L. Bovenberg and M. Raith, Monetary and Fiscal Policy Interaction and Government Debt Stabilization, Journal of Economics 62, 111-140, 1995.

[Ba89] Basar T., Time Consistency and Robustness of Equilibria in non-Cooperative Dynamic Games,

F. van der Ploeg and A.J. de Zeeuw (editors), Dynamic Policy Games in Economics, 9-54, North Holland, 1989.

[Ba95] Basar T. and G.J. Olsder, Dynamic Noncooperative Game Theory, Academic Press, London, 2nd edition, 1995.

[Bit97] Bitmead, R.R. and G. De Nicolao, Fake Riccati Equations for Stable Receding-Horizon Control, Proceedings ECC 97, Brussels, Conference ID 831, 1997.

[Eng98] Engwerda, J.C., On the Open-loop Nash Equilibrium in LQ-games, Journal of Economic Dynamics and Control 22, 729-762, 1998.

[Fe98] Feenstra, T.L., Environmental Policy Instruments and International Rivalry: a Dynamic Analysis,

PhD. Thesis, Tilburg University, The Netherlands, ISBN 905668 036 6, 1998.

[Le74] Leitmann, G.

Cooperative and Noncooperative Many Players Differential Games, Springer Verlag, Wien-New York, 1974.

[Ne95] Neck, R. and E.J. Dockner, Commitment and Coordination in a Dynamic Game Model of International Economic Policy-making, Open Economies Review 6, 5-28, 1995.

[Ni98] Nicolao, G. De, L. Magni and R. Scattolini, Stabilizing Receding-Horizon Control of Nonlinear Time-Varying Systems, IEEE Transactions on Automatic Control, 43(7), 1030-1036, 1998.

[Se91] Sethi, S. and G. Sorger, a Theory of Rolling Horizon Decision Making, Annals of Operation Research 29, 387-416, 1991.

[Ta86] Tabellini, G. Money, debt and deficits in a dynamic game, Journal of Economic Dynamics and Control 10, 427-442, 1986.

[Ze91] Zeeuw, A.J. de, and F. van der Ploeg, A Differential Game of International Pollution Control, System \& Control Letters 17, 409-414, 1991. 